\title{
Energy Levels and Atomic Lifetimes of Rydberg States in Neutral Indium
}

\author{
M. YILDIZ* \\ Department of Physics, Faculty of Kamil Ozdag Science, Karamanoglu Mehmetbey University \\ Campus, 70200 Karaman, Turkey \\ (Received December 22, 2011; revised version July 24, 2012; in final form September 25, 2012)
}

\begin{abstract}
Atomic lifetimes and energy levels were calculated using weakest bound potential model theory for $5 s^{2} n s^{2} S_{1 / 2}$ $(n \geq 6), 5 s^{2} n p^{2} P_{1 / 2}^{0}(n \geq 5), 5 s^{2} n p^{2} P_{3 / 2}^{0}(n \geq 5), 5 s^{2} n d^{2} D_{3 / 2}^{0}(n \geq 5), 5 s^{2} n d^{2} D_{5 / 2}^{0}(n \geq 5)$ series of the Rydberg states in neutral indium. The use of the quantum defect method and Martin's expression allowed us to supply lifetime values along by means of the series above. Some lifetimes and energy values not existing in the literature for high Rydberg states in neutral indium atom were obtained using this method. Our results nicely agree with the available experimental results and theoretical results.
\end{abstract}

DOI: 10.12693 /APhysPolA.123.25

PACS: $32.10 .-\mathrm{f}, 31.50 .-\mathrm{x}$

\section{Introduction}

The physical properties of highly excited Rydberg atoms such as the fine structure, the energy levels and the lifetimes of atomic Rydberg states, are of great importance in both theoretical and experimental research [1-6]. The determination of spectroscopic data for neutral and ionized atomic systems, particularly the high-lying Rydberg states, have been an active research area in astrophysics, laser physics, plasma physics, and thermonuclear fusion research $[7,8]$. During the last three decades, the most of researchers have studied the transition probabilities, energy levels, fine structure, lifetimes and oscillator strengths of excited states belonging to indium being in group-three elements having as ground state the $5 s^{2} 5 p$ configuration with three electrons outside the closed shell. Therefore, the two $s$ electrons form a closed sub-shell, the residual electron gives rise to an alkali-like spectrum [9-12]. The most of investigations in literature are limited few low excitations radiative lifetimes $(n \leq 8-12)$ [13].

Many of the modern experimental techniques and theoretical methods come across some difficulties in the exact measurement of the spectroscopic parameters such as transition probabilities, energy levels, fine structure, lifetimes, and oscillator strengths of many electron atoms and heavy ions. The physical parameters in highly lying Rydberg states are always difficult problems especially in theoretical studies because of indistinguishability of equivalent electrons and the necessity of taking into account many configurations or orbital basis-set functions [14]. It is impossible yet to solve many-electron systems without imposing severe approximations. Many theoretical methods exist for calculation of spectroscopic parameters for atomic or ionic systems

*e-mail: yildiz@kmu.edu.tr such as the Hartree-Fock approximations, configuration interaction methods, R-matrix methods, semi-empirical methods, and many-body perturbation theories.

In the present paper, we have calculated atomic lifetimes and energy levels of the Rydberg states in neutral indium using the weakest bound electron potential model theory (WBEPMT). The results for atoms having the principal quantum number up to $n=50$ were listed.

\section{Theoretical method}

WBEPMT, which was developed by Zheng et al., can be applied for determination of some physical parameters for example energy levels, ionization potentials, transition probabilities, oscillator strengths and lifetime of excited levels in many-electron atomic and ionic systems [15-22]. Zheng separated electrons into two groups: the weakest bound electron (WBE) and non-weakest bound electrons (NWBE), within a given system, to describe the electronic motion in multi-electron systems as a new model potential. The WBE in this systems can be excited or ionized easily. The WBEPM theory describes the WBE movement according to the potential field produced by the nucleus and the non-weakest bound electron. The behavior of WBE is affected by the one part of the potential field, dipole moment and the other part is the Coulomb potential. The introduction of $d$ effectively adjusts the integer quantum numbers $n$ and $l$ into non-integers $n^{*}$ and $l^{*}$; therefore, the principal quantum number $(n)$ and the angular momentum quantum number $(l)$ of the WBE is considered as the effective principal quantum number $\left(n^{*}\right)$ and effective angular momentum quantum number $\left(l^{*}\right)$. The investigation of behavior of WBE gives some valuable information about some atomic or ionic properties in multi-electron systems such as transition, excitation and ionization [17-22].

The Schrödinger equation of the weakest bound electron under non-relativistic approximation is given 
below $[15,17,18]$ :

$$
\begin{aligned}
& {\left[-\frac{1}{2} \nabla^{2}+V\left(r_{i}\right)\right] \psi_{i}=\varepsilon_{i} \psi_{i},} \\
& V\left(r_{i}\right)=-\frac{Z^{*}}{r_{i}}+\frac{[d(d+1)+2 d l]}{2 r_{i}^{2}} .
\end{aligned}
$$

The non-weakest bound electrons and nucleus produced potential function given as $V\left(r_{i}\right)$ in Eq. (2). $Z^{*}$ is the effective nuclear charge, $r_{i}$ is the distance between the weakest bound electron and the nucleus. In this theory, electronic radial wave functions are shown as a function of the Laguerre polynomial in terms of some parameters $[15,17,18]$ :

$$
\begin{aligned}
& R=\left(\frac{2 Z^{*}}{n^{*}}\right)^{l^{*}+3 / 2}\left[\frac{2 n^{*}}{\left(n^{*}-l^{*}-1\right) !} \Gamma\left(n^{*}-l^{*}+1\right)\right]^{-1 / 2} \\
& \quad \times \exp \left(-\frac{Z^{*} r}{n^{*}}\right) r^{l^{*}} L_{n^{*}-l^{*}-1}^{2 l^{*}+1}\left(\frac{2 Z^{*} r}{n^{*}}\right) \\
& n^{*} \text { and } l^{*} \text { parameters are described as } \\
& n^{*}=n+d \text { and } l^{*}=l+d .
\end{aligned}
$$

The energy expression of weakest bound electron is shown as

$$
\varepsilon=-\frac{Z^{* 2}}{2 n^{* 2}},
$$

where both $Z^{*}$ and $d$ are unknown parameters. This complexity could be reduced by making Eq. (5) similar to quantum defect theory. The energy expression in the quantum defect theory is demonstrated in Eq. (6) [23]:

$$
\varepsilon=-\frac{Z_{\text {net }}{ }^{2}}{2 n^{* 2}},
$$

where $n^{*}=n-\delta, \delta$ is quantum defect and constant for a given fixed orbital quantum number and $Z_{\text {net }}$ is the ion core charge. For a neutral atom, the value of a neutral atom $Z_{\text {net }}$ is 1 and it is 2 for a singly charged ion $Z_{\text {net }}$ For neutral atoms, energy eigenvalue can be introduced as

$$
\varepsilon=-\frac{1}{2(n-\delta)^{2}},
$$

where $n$ is the principal quantum number and $\delta$ can be determined by Martin's expression (Eq. (8)) [24]:

$$
\delta=a+b\left(n-\delta_{0}\right)^{-2}+c\left(n-\delta_{0}\right)^{-4}+d\left(n-\delta_{0}\right)^{-6},
$$

where $\delta_{0}$ is the constant given in the Rydberg series as quantum defect of lowest energy state. Coefficients $a ; b ; c ; d$ can be calculated from the first four experimental values by solving Eq. (7) and Eq. (8). Extrapolating with these values could provide calculation of the energy levels of high Rydberg states. This formula has been applied to sodium atom for analysis of spectra and it has resulted in high accurate outcome. Rykova's expressions given below can be used to calculate the lifetimes of excited levels for many electron atomic systems [25]:

$$
\tau=\tau_{0}\left(n^{*}\right)^{\alpha} \text {. }
$$

$\tau_{0}$ and $\alpha$ are coefficients in relevant series and can be calculated from the experimental values of energy and lifetime described in the WBEPM theory.

\section{Results and discussions}

The present study reports the calculation of energy levels and atomic lifetimes using weakest bound electron potential model theory for $5 s^{2} n s^{2} S_{1 / 2}(n \geq 6), 5 s^{2} n p$ ${ }^{2} P_{1 / 2}^{0}(n \geq 5), 5 s^{2} n p^{2} P_{3 / 2}^{0}(n \geq 5), 5 s^{2} n d{ }^{2} D_{3 / 2}^{0}(n \geq$ $5), 5 s^{2} n d^{2} D_{5 / 2}^{0}(n \geq 5)$ series of the Rydberg states in neutral indium.

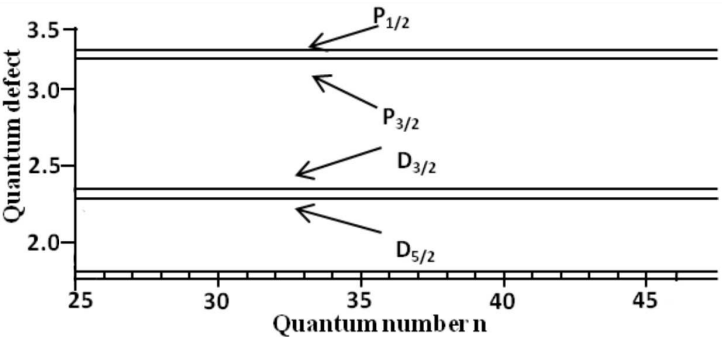

Fig. 1. Quantum defects of Rydberg series as a function of the quantum number [28, 29].

The parameters required for the calculations of energy levels and atomic lifetimes was determined using the procedure mentioned above and the coefficients $a, b, c, d$ in Eq. (8) and the values of $\delta_{0}$ belonging to different series were fitted to experimental energy values [26] in the indium atom (Tables I, II). The obtained $\delta_{0}$ quantum defect of lowest energy state $5 s^{2} n p^{2} P_{1 / 2}^{0}(n \geq 5), 5 s^{2} n p{ }^{2} P_{3 / 2}^{0}$ $(n \geq 5), 5 s^{2} n d^{2} D_{3 / 2}^{0}(n \geq 5), 5 s^{2} n d^{2} D_{5 / 2}^{0}(n \geq 5)$ Rydberg series was compared and the agreement with the current literature (Fig. 1) has been observed [12].

TABLE II

The coefficients of lifetime for neutral indium.

\begin{tabular}{c|c|c}
\hline \hline Series & $\tau_{0}$ & $\alpha$ \\
\hline $5 s^{2} n s^{2} S_{1 / 2}(n \geq 6)$ & 0.372132 & 3.013465 \\
$5 s^{2} n p^{2} P_{1 / 2}^{0}(n \geq 5)$ & 1.556491 & 3.722339 \\
$5 s^{2} n p^{2} P_{3 / 2}^{0}(n \geq 5)$ & 1.461889 & 3.651926 \\
$5 s^{2} n d^{2} D_{3 / 2}^{0}(n \geq 5)$ & 0.919853 & 2.554778 \\
$5 s^{2} n d^{2} D_{5 / 2}^{0}(n \geq 5)$ & 0.421144 & 3.004073
\end{tabular}

Figure 1 displays the calculated quantum defects $\delta_{n}$ of ${ }^{2} P_{3 / 2,1 / 2}$ and ${ }^{2} D_{3 / 2,5 / 2}$ series as a function of $n$. It is known that in the ${ }^{2} P_{3 / 2,1 / 2}$ and ${ }^{2} D_{3 / 2,5 / 2}$ series, $\delta_{n}$ of the $J=l+1 / 2$ are larger than that of the $J=l-1 / 2$. It is compatible with the obtained $\delta_{0}$ quantum defect of these series. In addition $\delta_{0}$ quantum defect of lowest energy state $5 s^{2} n s^{2} S_{1 / 2}(n \geq 6)$ was given in Table I. $\tau_{0}$ and $\alpha$ coefficients have been calculated using the WBEPM theory. Let us note that $\alpha$ coefficients in Table II are approximately around three. Because in high Rydberg series the lifetimes are expected to be proportional to a power, around three, of the effective principal quantum number. The values of energy levels and atomic lifetimes have been obtained using these parameters and have been presented in Tables I, II. 
Spectral coefficients of energy level series for neutral indium.

TABLE I

\begin{tabular}{c|c|c|c|c|c}
\hline \hline Series & $a$ & $b$ & $c$ & $d$ & $\delta_{0}$ \\
\hline $5 s^{2} n s^{2} S_{1 / 2}(n \geq 7)$ & 2.720569 & 0.244069 & 0.394892 & -0.588441 & 2.781528 \\
$5 s^{2} n p^{2} P_{1 / 2}^{0}(n \geq 5)$ & 3.223092 & 0.351695 & 0.652420 & -0.313019 & 3.466590 \\
$5 s^{2} n p^{2} P_{3 / 2}^{0}(n \geq 6)$ & 3.197460 & 0.319445 & 0.925562 & -0.825711 & 3.421306 \\
$5 s^{2} n d^{2} D_{3 / 2}^{0}(n \geq 5)$ & 2.496426 & -12.832927 & 197.257055 & -917.991306 & 2.177824 \\
$5 s^{2} n d^{2} D_{5 / 2}^{0}(n \geq 5)$ & 2.415336 & -9.027419 & 131.879455 & -599.356134 & 2.175465
\end{tabular}

TABLE III

Comparison between the calculated and experimental values of energy level and lifetime for $\operatorname{In}(\mathrm{I}): 5 s^{2} n s^{2} S_{1 / 2}(n \geq 6)$.

\begin{tabular}{|c|c|c|c|c|c|c|c|}
\hline$n$ & $\begin{array}{c}E_{\exp }\left[\mathrm{cm}^{-1}\right] \\
\text { Ref. }[26]\end{array}$ & $\begin{array}{c}E_{\text {cal }}\left[\mathrm{cm}^{-1}\right] \\
\text { this work }\end{array}$ & $\tau[\mathrm{ns}]$ & $\begin{array}{c}\text { Exp. results } \\
\tau \text { [ns] Ref. [30] }\end{array}$ & $\begin{array}{c}\text { Exp. results } \\
\tau \text { [ns] Ref. [10] }\end{array}$ & $\begin{array}{c}\text { SD results } \\
\tau \text { [ns] Ref. [9] }\end{array}$ & $\begin{array}{c}\text { Exp. results } \\
\tau \text { [ns] Refs. }[31,32]\end{array}$ \\
\hline 6 & 22297 & 22297 & 7 & & $7.5 \pm 0.7$ & 7.04 & \\
\hline 7 & 10368 & 10368 & 29 & $27 \pm 6$ & & 21.5 & $19.5 \pm 1.5$ \\
\hline 8 & 6033 & 6033 & 56 & $55 \pm 6$ & & 47.7 & $53 \pm 5$ \\
\hline 9 & 3951 & 3951 & 94 & $104 \pm 12$ & & 89.4 & $118 \pm 10$ \\
\hline 10 & 2789 & 2788 & 147 & $163 \pm 13$ & & & \\
\hline 11 & 2074 & 2070 & 217 & $244 \pm 19$ & & & \\
\hline 12 & 1602 & 1600 & 306 & $330 \pm 21$ & & & \\
\hline 13 & 1275 & 1274 & 417 & $490 \pm 35$ & & & \\
\hline 14 & 1039 & 1038 & 551 & $625 \pm 60$ & & & \\
\hline 15 & 863 & 862 & 712 & $785 \pm 785$ & & & \\
\hline 16 & 728 & 727 & 902 & $1025 \pm 70$ & & & \\
\hline 17 & 622 & 622 & 1122 & $1170 \pm 95$ & & & \\
\hline 18 & 538 & 538 & 1377 & $1360 \pm 135$ & & & \\
\hline 19 & 470 & 470 & 1666 & $1690 \pm 200$ & & & \\
\hline 20 & 414 & 414 & 1995 & $2000 \pm 300$ & & & \\
\hline 30 & 368 & 367 & 7898 & & & & \\
\hline 40 & & 147 & 20242 & & & & \\
\hline 50 & & 78 & 41425 & & & & \\
\hline
\end{tabular}

Tables III-VII present the comparison with the experimental and theoretical data given in the literature. It can be seen that experimental and theoretical studies with energy levels and lifetimes of atomic indium were insufficient in the literature. In this paper, atomic lifetimes and energy levels of $5 s^{2} n s^{2} S_{1 / 2}(n \geq 6), 5 s^{2} n p{ }^{2} P_{1 / 2}^{0}$ $(n \geq 5), 5 s^{2} n p{ }^{2} P_{3 / 2}^{0}(n \geq 5), 5 s^{2} n d{ }^{2} D_{3 / 2}^{0}(n \geq 5)$, $5 s^{2} n d^{2} D_{5 / 2}^{0}(n \geq 5)$ series of the Rydberg states in neutral indium were calculated and listed here for relevant series of indium atom having the principal quantum number up to $n=50$, though they were given up to $n=9$ in the literature. The energy levels and lifetimes in both lower lying and highly excited Rydberg states have great importance in the many areas of the physics. Therefore, our energy levels and lifetimes results obtained from the WBEPM theory have been compared with relativistic many-body perturbation theory (RMBPT) and all-order single-double (SD) method results given by Safronova et al. [9], results in NIST [26], the results of Andersen and Sorensen including experimental data [10] and the experimental results of Jönsson et al. [30].

Safronova et al. [9] used relativistic many-body perturbation theory and all-order SD method to study en- ergies, lifetimes like other physical properties of indium. The calculations made by Safronova et al. allow one to study convergence of perturbation theory and estimate the uncertainty of theoretical predictions. Andersen and Sorensen [10] used the beam foil technique that presents an ideal tool for systematic study but the beam foil technique has certain limitations. It is difficult to study the Rydberg states using this method. Andersen et al. studied excited levels with low main quantum numbers more than excited levels with high main quantum numbers. The intensity of the Rydberg states is small and lifetimes relatively long. Jönsson et al. [30] have measured radiative lifetimes of some sequences of indium using pulsed laser excitation of an atomic beam. They used UV pulses produced by a YAG-pumped or excimer-pumped dye-laser system. The results obtained from the lifetime measurements for the lower lying states are proportional to a power, close to three, of the effective principal quantum number. Ewiss et al. measured the natural lifetimes in $\operatorname{In}(\mathrm{I})(n \leq 8-12)$ using fluorescence decay [31, 32]. 
TABLE IV

Comparison between the calculated and experimental values of energy level and lifetime for $\operatorname{In}(\mathrm{I}): 5 s^{2} n d^{2} D_{3 / 2}(n \geq 5)$.

\begin{tabular}{|c|c|c|c|c|c|c|}
\hline$n$ & $\begin{array}{c}E_{\exp }\left[\mathrm{cm}^{-1}\right] \\
\text { (Ref. }[26])\end{array}$ & $\begin{array}{c}E_{\text {cal }}\left[\mathrm{cm}^{-1}\right] \\
\text { this work }\end{array}$ & $\begin{array}{c}\text { This work } \\
\tau \text { [ns] }\end{array}$ & $\begin{array}{c}\text { Exp. results } \\
\tau \text { [ns] (Ref. [30]) }\end{array}$ & $\begin{array}{c}\text { Exp. results } \\
\tau \text { [ns] (Ref. [10]) }\end{array}$ & $\begin{array}{c}\text { SD results } \\
\tau \text { [ns] (Ref. [9]) }\end{array}$ \\
\hline 5 & 13778 & 13778 & 9 & & $6.3 \pm 0.5$ & 6.45 \\
\hline 6 & 7809 & 7809 & 22 & & $21 \pm 3$ & 19.2 \\
\hline 7 & 4834 & 4834 & 42 & $200 \pm 4$ & $50 \pm 5$ & 42 \\
\hline 8 & 3334 & 3334 & 71 & $317 \pm 22$ & & 75.7 \\
\hline 9 & 2436 & 2594 & 109 & $550 \pm 50$ & & \\
\hline 10 & 1855 & 1949 & 158 & $455 \pm 40$ & & \\
\hline 11 & 1459 & 1517 & 218 & $490 \pm 50$ & & \\
\hline 12 & 1177 & 1215 & 289 & $485 \pm 40$ & & \\
\hline 13 & 968 & 994 & 374 & $500 \pm 30$ & & \\
\hline 14 & 811 & 829 & 471 & $570 \pm 40$ & & \\
\hline 15 & 693 & 701 & 583 & $635 \pm 40$ & & \\
\hline 16 & 595 & 601 & 710 & $735 \pm 60$ & & \\
\hline 17 & 512 & 521 & 853 & $820 \pm 65$ & & \\
\hline 18 & 449 & 456 & 1011 & $895 \pm 60$ & & \\
\hline 19 & 399 & 402 & 1186 & $1075 \pm 70$ & & \\
\hline 20 & 355 & 358 & 1379 & $1275 \pm 115$ & & \\
\hline 30 & 145 & 145 & 4375 & & & \\
\hline 40 & & 78 & 9663 & & & \\
\hline 50 & & 48 & 17675 & & & \\
\hline
\end{tabular}

TABLE V

Comparison between the calculated and experimental values of energy level and lifetime for $\operatorname{In}(\mathrm{I}): 5 s^{2} n p^{2} P_{1 / 2}(n \geq 5)$.

\begin{tabular}{|c|c|c|c|c|}
\hline$n$ & $E_{\exp }\left[\mathrm{cm}^{-1}\right]$ (Ref. [26]) & $E_{\text {cal }}\left[\mathrm{cm}^{-1}\right]$ this work & This work $\tau$ [ns] & SD results $\tau$ [ns] (Ref. [9]) \\
\hline 5 & 46670 & 46670 & 13 & \\
\hline 6 & 14853 & 14853 & 69 & 69.7 \\
\hline 7 & 7809 & 7809 & 218 & 219 \\
\hline 8 & 4843 & 4843 & 525 & 473 \\
\hline 9 & 3301 & 3288 & 1065 & \\
\hline 10 & 2396 & 2389 & 1929 & \\
\hline 11 & 1817 & 1814 & 3221 & \\
\hline 12 & & 1424 & 5053 & \\
\hline 13 & & 1148 & 7551 & \\
\hline 14 & & 1148 & 10850 & \\
\hline 15 & & 791 & 15096 & \\
\hline 16 & & 672 & 20447 & \\
\hline 17 & & 578 & 27068 & \\
\hline 18 & & 578 & 35134 & \\
\hline 19 & & 440 & 44832 & \\
\hline 20 & & 389 & 56356 & \\
\hline 30 & & 153 & 321185 & \\
\hline 40 & & 81 & 1046525 & \\
\hline 50 & & 50 & 2560953 & \\
\hline
\end{tabular}

While the calculation procedure for the systems with a few electrons can be carried out easily, the calculations become more difficult and complex in the case of increasing number of electrons. Especially, for the excited states and the Rydberg states of many-electron systems, more configurations must be considered. Therefore, calculations become more complicated. 
TABLE VI

Comparison between the calculated and experimental values of energy level and lifetime for $\operatorname{In}(\mathrm{I}): 5 s^{2} n p^{2} P_{3 / 2}(n \geq 5)$.

\begin{tabular}{|c|c|c|c|c|c|}
\hline$n$ & $\begin{array}{c}E_{\exp }\left[\mathrm{cm}^{-1}\right] \\
\text { (Ref. }[26])\end{array}$ & $\begin{array}{c}E_{\text {cal }}\left[\mathrm{cm}^{-1}\right] \\
\text { this work } \\
\end{array}$ & $\begin{array}{c}\text { This work } \\
\tau[\mathrm{ns}]\end{array}$ & $\begin{array}{c}\text { SD results } \\
\tau \text { [ns] (Ref. [9]) }\end{array}$ & $\begin{array}{c}\text { Exp. results } \\
\tau \text { [ns] (Refs. [31, 32]) }\end{array}$ \\
\hline 5 & 44031 & 44031 & 12 & \multirow{19}{*}{$\begin{array}{c}63.7 \\
192 \\
414\end{array}$} & \multirow{19}{*}{$55 \pm 4$} \\
\hline 6 & 14555 & 14555 & 63 & & \\
\hline 7 & 7697 & 7697 & 192 & & \\
\hline 8 & 4789 & 4789 & 450 & & \\
\hline 9 & 3271 & 3259 & 898 & & \\
\hline 10 & 2376 & 2371 & 1606 & & \\
\hline 11 & 1805 & 1802 & 2650 & & \\
\hline 12 & & 1416 & 4116 & & \\
\hline 13 & & 1142 & 6098 & & \\
\hline 14 & & 940 & 8695 & & \\
\hline 15 & & 787 & 12014 & & \\
\hline 16 & & 669 & 16168 & & \\
\hline 17 & & 576 & 21279 & & \\
\hline 18 & & 500 & 27472 & & \\
\hline 19 & & 439 & 34880 & & \\
\hline 20 & & 388 & 43640 & & \\
\hline 30 & & 152 & 240164 & & \\
\hline 40 & & 81 & 764514 & & \\
\hline 50 & & 50 & 1839146 & & \\
\hline
\end{tabular}

TABLE VII

Comparison between the calculated and experimental values of energy level and lifetime for $\operatorname{In}(\mathrm{I}): 5 s^{2} n d^{2} D_{5 / 2}(n \geq 5)$.

\begin{tabular}{|c|c|c|c|c|c|c|c|}
\hline$n$ & $\begin{array}{c}E_{\exp }\left[\mathrm{cm}^{-1}\right] \\
\text { (Ref. [26]) }\end{array}$ & $\begin{array}{c}E_{\mathrm{cal}}\left[\mathrm{cm}^{-1}\right] \\
\text { this work }\end{array}$ & $\begin{array}{c}\text { This work } \\
\tau \text { [ns] }\end{array}$ & $\begin{array}{c}\text { Exp. results } \\
\tau \text { [ns] } \\
\text { (Ref. [30]) }\end{array}$ & $\begin{array}{c}\text { Exp. results } \\
\tau[\mathrm{ns}] \\
\text { (Ref. [10]) }\end{array}$ & $\begin{array}{c}\text { SD results } \\
\quad \tau \text { [ns] } \\
\text { (Ref. [9]) }\end{array}$ & $\begin{array}{c}\text { Exp. results } \\
\tau \text { [ns] } \\
\text { (Refs. }[31,32])\end{array}$ \\
\hline 5 & 13755 & 13755 & 7 & & $7.6 \pm 0.5$ & 6.78 & \\
\hline 6 & 7697 & 7697 & 20 & & $22 \pm 3$ & 20.1 & $18.6 \pm 1.5$ \\
\hline 7 & 4808 & 4808 & 45 & $147 \pm 10$ & $50 \pm 5$ & 44.0 & $154 \pm 10$ \\
\hline 8 & 3315 & 3315 & 87 & $238 \pm 20$ & & 77.2 & $300 \pm 60$ \\
\hline 9 & 2421 & 2530 & 151 & & & & \\
\hline 10 & 1843 & 1907 & 242 & & & & \\
\hline 11 & 1449 & 1489 & 365 & & & & \\
\hline 12 & 1468 & 1194 & 527 & & & & \\
\hline 13 & 961 & 979 & 733 & & & & \\
\hline 14 & 805 & 817 & 990 & & & & \\
\hline 15 & 688 & 692 & 1304 & & & & \\
\hline 16 & 591 & 594 & 1681 & & & & \\
\hline 17 & 509 & 515 & 2129 & & & & \\
\hline 18 & 447 & 451 & 2653 & & & & \\
\hline 19 & 397 & 398 & 3263 & & & & \\
\hline 20 & 353 & 354 & 3963 & & & & \\
\hline 30 & 144 & 144 & 17693 & & & & \\
\hline 40 & & 77 & 49452 & & & & \\
\hline 50 & & 48 & 108296 & & & & \\
\hline
\end{tabular}

Because of the difficulties mentioned above, the theoretical and experimental studies generally consider low lying states rather than highly excited states. It can be seen from the tables that our results are very close to the corresponding theoretical and experimental results. These results prove that Martin's expression is convenient for the Rydberg series of neutral indium. The WBEPM theory has a simple calculation procedure. It 
can be used to calculate the lifetimes and energy levels for both highly excited states and low lying states without any increase in complexity in calculation process. Previously, many of spectroscopic data such as transition probabilities, oscillator strengths, lifetimes of excited levels and ionization energies were obtained using the WBEPM theory in many-electron atomic and ionic systems [33-38]. The semi-empirical methods such as the WBEPM theory where one or more parameters needs to be adjusted according to the existing experimental data can be considered as a useful method for much more complicated systems, for especially highly excited states.

In this study, by courtesy of this method, we have calculated the energy levels and lifetimes belonging to higher excited levels than published in the literature for neutral indium.

\section{Acknowledgments}

The author gratefully acknowledges Gültekin Çelik, Yasin Gökçe, and Şule Ateş for their help and for the support of the University of Karamanoglu Mehmetbey Scientific Research Projects (BAP) Coordinating Office.

\section{References}

[1] N.H. Linder, A. Peres, D.R. Terno, Phys. Rev. A 68, 042308 (2003)

[2] N.N. Nedeljkovic, L.D. Nedeljkovic, M.A. Mirkovic, Phys. Rev. A 68, 012721 (2003)

[3] J.Z. Zhang, Phys. Rev. Lett. 93, 043002 (2004).

[4] T.F. Gallagher, Rydberg Atoms, Cambridge University, Cambridge 1994.

[5] S. Feneuille, P. Jacquinot, Adv. At. Mol. Phys. 17, 99 (1981).

[6] J.A.C. Gallas, G. Leuchs, H. Walther, H. Figger, Adv. At. Mol. Phys. 20, 413 (1985).

[7] E. Biemont, P. Palmeri, P. Quinet, Z. Dai, S. Swanberg, H.L. Xu, J. Phys. B At. Mol. Opt. Phys. 38, 3547 (2005)

[8] W.O. Younis, S.H. Allam, Th.M.E. Sherbini, At. Data Nucl. Data Tables 187, 205 (2006).

[9] U.I. Safronova, M.S. Safronova, M.G. Kozlov, Phys. Rev. A 76, 022501 (2007)

[10] T. Andersen, G. Sorensen, Phys. Rev. A 5, 2447 (1972)

[11] M. Norton, A. Gallagher, Phys. Rev. A 3, 915 (1971).

[12] F.L. Hong, H. Maeda, Y. Matsuo, M. Takami, Phys. Rev. A 51, 1994 (1995).

[13] M. Yıldız, G. Çelik, H.Ş. Kılıç, Acta Phys. Pol. A 115, 641 (2009).
[14] L.L. Shimon, N.M. Erdevdi, Opt. Spectrosc. 42, 137 (1977).

[15] N.W. Zheng, A New Outline of Atomic Theory, JiangSu Education Press, Nanjing 1988.

[16] N.W. Zheng, Chin. Sci. Bull. 33, 916 (1988).

[17] N.W. Zheng, T. Wang, D.X. Ma, T. Zhou, J. Fan, Int. J. Quant. Chem. 98, 281 (2004).

[18] N.W. Zheng, T. Wang, Chem. Phys. 282, 31 (2002).

[19] N.W. Zheng, T. Wang, R.Y. Yang, J. Chem. Phys. 113, 6169 (2000).

[20] N.W. Zheng, T. Wang, T. Zhou, D.X. Ma, J. Phys. Soc. Jpn. 71, 1672 (2002).

[21] N.W. Zheng, Y.J. Sun, D.X. Ma, R. Yang, T. Zhou, T. Wang, Int. J. Quant. Chem. 81, 232 (2001).

[22] N.W. Zheng, T. Wang, Astrophys. J. Suppl. Ser. 143, 231 (2002).

[23] D.R. Bates, A. Damgaard, Philos. Trans. A 242, 101 (1949).

[24] W.C. Martin, J. Opt. Soc. Am. 70, 784 (1980).

[25] O.V. Rykova, Y.F. Veroalienen, Opt. Spectrosc. 76, 23 (1994).

[26] Y. Ralchenko, F.-C. Jou, D.E. Kelleher, A.E. Kramida, A. Musgrove, J. Reader, W.L. Wiese, K. Olsen, NIST Atomic Spectra Database (version 3.0.2), [Online]. National Institute of Standards and Technology, 2005.

[27] N.W. Zheng, Z. Li, D.X. Ma, T. Zhou, J. Fan, Canad. J. Phys. 82, 523 (2004)

[28] J.H.M. Neijzen, A. Dönszelmann, Physica $C$ 106, 271 (1981).

[29] J.H.M. Neijzen, A. Dönszelmann, Physica C 111, 127 (1981).

[30] G. Jönsson, H. Lundeberg, S. Svanberg, Phys. Rev. A 27, 2935 (1983).

[31] M.A.Z. Ewiss, C. Sonek, J. Phys. B 16, L153 (1983).

[32] M.A.Z. Ewiss, C. Sonek, A. Dönszelmann, Astron. Astrophys. 121, 327 (1983).

[33] G. Çelik, M. Yıldız, H.Ş. Kılıç, Acta Phys. Pol. A 112, 485 (2007).

[34] G. Celik, J. Quant. Spectrosc. Radiat. Transfer 103. 578 (2007)

[35] G. Çelik, E. Akın, H.Ş. Kılıç, Eur. Phys. J. D 40, 325 (2006)

[36] G. Çelik, Ş. Ateş, Eur. Phys. J. D 44, 433 (2007)

[37] Ş. Ateş, G. Tekeli, G. Çelik, M. Akın, M. Taşer, Eur. Phys. J. D 54, 21 (2009).

[38] G. Çelik, E. Akın, H.Ş. Kılıç, Int. J. Quant. Chem. 107, 495 (2007). 